\title{
Overexpression of sialidase NEU3 increases the cellular radioresistance potential of U87MG glioblastoma cells
}

\author{
Flavia Orizio a, Luca Triggiani ${ }^{\mathrm{b}}$, Antonella Colosini ${ }^{\mathrm{a}}$, Michela Buglione ${ }^{\mathrm{b}}$, Nadia Pasinetti ${ }^{\mathrm{b}}$, \\ Eugenio Monti ${ }^{\text {a }}$, Roberto Bresciani ${ }^{\text {a, * }}$ \\ a Department of Molecular and Translational Medicine, Unit of Biotechnology, University of Brescia, Viale Europa 11, 25123, Brescia, Italy \\ ${ }^{\mathrm{b}}$ Department of Radiation Oncology, University of Brescia and Spedali Civili Hospital, Brescia, Piazzale Spedali Civili 1, 25123, Brescia, Italy
}

\section{A R T I C L E I N F O}

Article history:

Received 31 October 2018

Accepted 13 November 2018

Available online xxx

\section{Keywords:}

Sialidase

NEU3

Glioblastoma

U87MG cells

Radioresistance

PI3K/Akt

\begin{abstract}
A B S T R A C T
The plasma membrane-associated sialidase NEU3 is known to play important roles in different physiological and pathophysiological processes such as proliferation, cellular differentiation and tumorigenesis. Up-regulation of NEU3 has been associated to several tumors and recently it was demonstrated that its down-modulation in glioblastoma cells promotes cell invasiveness. To date, no information concerning the possible role played by NEU3 in relation to tumor radioresistance is available. Here we show that overexpression of NEU3 in glioblastoma U87MG cells activates PI3K/Akt signaling pathway resulting in an increased radioresistance capacity and in an improved efficiency of double strand DNA-repair mechanisms after irradiation. Our results demonstrate for the first time that NEU3 contributes to the radioresistance features of U87MG cells, bringing to evidence a novel rand peculiar role of the enzyme in cancer biology.
\end{abstract}

๑) 2018 Elsevier Inc. All rights reserved.

\section{Introduction}

Sialidases or neuraminidases (E.C. 3.2.1.18) are exoglycosidases that play a pivotal role in sialoglycoconjugates metabolism and in cell growth and cellular differentiation [1]. These enzymes remove sialic acid residues from the terminal ends of the oligosaccharide chains of glycoconjugates. Among the four different mammalian sialidases (NEU1-NEU4) identified [2-4], NEU3 is the plasma membrane-associated form of the enzyme, present at the cell surface and associated to the external leaflet of the plasma

\footnotetext{
* Corresponding author. Department of Molecular and Translational Medicine, University of Brescia, Viale Europa 11, 25123, Brescia, Italy.

E-mail addresses: flavia.orizio88@gmail.com (F. Orizio), luca.triggiani@unibs.it (L. Triggiani), anto.colo90@libero.it (A. Colosini), michela.buglione@unibs.it (M. Buglione), nadia_pasinetti@yahoo.it (N. Pasinetti), eugenio.monti@unibs.it (E. Monti), roberto.bresciani@unibs.it (R. Bresciani).

1 The abbreviations used are: Ganglioside GM3, NeuAc $\alpha 2-3$ Gal $\beta 1-4$ Glc $\beta-C e r$; Gangliosides GD1a, NeuAc $\alpha 2-3 G$ al $\beta 1-3 G a l N A c \beta 1-4(N e u A c \alpha 2-3) G a l \beta 1-4 G l c \beta-C e r ;$ GBM, glioblastoma multiforme; HA, human influenza hemagglutinin; mTORC, mammalian Target of Rapamycin Complex; PI3K, phosphatidylinositide 3-kinase; Gy, Gray; ATM, Ataxia Telangiectasia Mutated protein kinase; $\gamma \mathrm{H} 2 \mathrm{AX}$, phosphorylated H2AX histone; DNA-DSB, DNA double strand brakes; DDR, DNA Damage Response; DNA-PK, DNA-dependent Protein Kinase; PDK1, phosphatidylinositidedependent kinase- 1 .
}

membrane [5] and exosomes [6]. Gangliosides, particularly GM3 ${ }^{1}$ and GD1a, are the preferential substrates of the enzyme [7-9]. By modifying the cellular ganglioside composition, NEU3 regulates different physiological phenomena like proliferation, apoptosis but also tumor transformation [10,11]. Up-regulation of NEU3 has been correlated to different human tumors such as colon, renal, prostate and ovarian cancers [12]. Overexpression of NEU3 in HeLa cells induces the phosphorylation of Akt even in the absence EGF [13] and a recent study has demonstrated that NEU3 can also directly act on EGFR glycan(s), resulting in the activation of the receptor [14]. In the last few years many scientific papers demonstrated the involvement of the enzyme in oncogenic transformation mediated by EGFR $[10,15]$. Amplification of EGFR gene, that results in EGFR overexpression, is the most frequent alteration in Glioblastoma Multiforme (GBM) [16], that represents the most common and aggressive primary brain tumor [17]. To date, standard therapy for GBM includes maximal resection followed by radiotherapy and concomitant adjuvant chemotherapy [18]. Nevertheless, survival after diagnosis varies from 12 to 15 months, with less than $3-5 \%$ of patients surviving beyond five years [19].

In this study, we analyzed whether expression of sialidase NEU3 could influence the radioresistance characteristics of the GBM cell line U87MG. 


\section{Material and methods}

\subsection{Chemicals and reagents}

All chemicals are molecular biology-grade (SIGMA-Aldrich) unless specified. Cetuximab (MerckSerono) was kindly provided by Dr. L. Triggiani from Istituto del Radio "O. Alberti", Department of Radiation Oncology, University of Brescia and Spedali Civili Hospital, Brescia.

\subsection{Cell culture, transfection and treatments}

U87MG cells were obtained from IZSLER (Istituto Zooprofilattico Sperimentale della Lombardia e dell'Emilia Romagna, Brescia, Italy) and cultured in DMEM (GIBCO) containing $4 \mathrm{mM}$ L-Glutamine, 100 Units $/ \mathrm{ml}$ Penicillin, $100 \mu \mathrm{g} / \mathrm{ml}$ Streptomycin and 10\% (v/v) Fetal Bovine Serum (FBS) (GIBCO). Cells were stably transfected with pcDNA3.1-mNEU3-HA [13], while Mock cells were obtained by transfection with pcDNA3.1 empty vector. Transfectants were grown in complete medium supplemented with $0.5 \mathrm{mg} / \mathrm{ml} \mathrm{G418.}$ Treatment with inhibitors was performed by adding to the medium $5 \mu \mathrm{M}$ Erlotinib (Cell Signaling) for $1 \mathrm{~h}$; $60 \mathrm{nM}$ Cetuximab (MerckSerono) for $1 \mathrm{~h}$; $50 \mu \mathrm{M}$ LY-2094002 (Cell Signaling) for $1 \mathrm{~h} ; 5 \mu \mathrm{M}$ KU-60019 (Selleckem) for 24 h; $5 \mu$ M KU-0063794 (Selleckem) for $24 \mathrm{~h}$.

\subsection{Protein determination and sialidase activity assay}

Protein determination was performed using the Bredford Method Coomassie Protein Assay Reagent. The enzymatic activity was determined as described previously [9] using $0.1 \mathrm{mM} \mathrm{4-MU-}$ NeuAc as substrate. One Unit of sialidase activity is defined as the liberation of $1 \mu \mathrm{mol}$ of NeuAc $\min ^{-1}$ at $37^{\circ} \mathrm{C}$.

\subsection{Ionizing radiation}

Cells were irradiated at the Istituto del Radio "O. Alberti" Department of Radiation Oncology, University and Spedali Civili Hospital, Brescia - using a Linac Accelerator (ELekta SL75), nominal Energy $6 \mathrm{MV}$. Irradiation was performed with gantry angle $180^{\circ}$ and a $20 \times 20 \mathrm{~cm}$ field was used. The culture dishes were on top of a $3 \mathrm{~cm}$ plexiglass block and were positioned in correspondence of the isocenter plane (Source-Dish distance $100 \mathrm{~cm}$ ). Cells were exposed to a single radiation dose of 0,2 or $4 \mathrm{~Gy}$ and then incubated at $37^{\circ} \mathrm{C}$. For Western Blot analysis and clonogenic assay cells were collected 30 min from irradiation. For immunofluorescence analysis cells were fixed at established time intervals after radiation treatment.

\subsection{Clonogenic assay}

Cells were grown in complete DMEM and treated with different radiation doses. After $30 \mathrm{~min}$ cells were collected by scraping, resuspended in cold culture medium and seeded for clonogenic cell survival assay and the remaining cells were used for Western-blot analysis. Cells were grown in complete DMEM for 14 days. Surviving colonies were fixed with $80 \%$ ice cold ethanol and stained with $0.1 \%$ Crystal Violet in $20 \%$ methanol. Only colonies of 50 cells or more were counted. Surviving fractions were calculated as the ratio between the colonies formed by irradiated cells and those formed by non-irradiated cells.

\subsection{Immunoblotting}

Proteins were separated by SDS/10\% PAGE and transferred to a Hybond-P PVDF membrane (GE Healthcare). Membranes were then blocked with 5\% (w/v) BSA Cohn fraction V in PBS, washed with PBS containing $0.1 \%$ Tween 20 (PBST) and incubated with primary antibody diluted in PBST containing $1 \%(\mathrm{w} / \mathrm{v})$ BSA for $1 \mathrm{~h}$ at room temperature. After washes with PBST, membranes were incubated with HRP-conjugated secondary antibody diluted in PBST. Immunocomplexes were detected using the Western-blot Luminol Reagent (Santa Cruz). The following primary antibodies were used: rabbit anti-HA (1:1000; Sigma); mouse anti-GAPDH (1:8000; Millipore); rabbit anti-phospho-ERK1/2 (1:500; Cell Signaling); rabbit anti-ERK1/2 (1:500; Cell Signaling); rabbit anti-phospho-AKT Ser473 (1:1000; Cell Signaling); rabbit anti-phospho-AKT Thr308 (1:1000; Cell Signaling); rabbit anti-AKT (1:1000; Cell Signaling). The following secondary antibodies were used: goat anti-rabbit (1:5000; Cell Signaling) and donkey ant-mouse (1:10000; Sigma).

\subsection{Immunofluorescence}

Cells were seeded onto glass coverslips and after $48 \mathrm{~h}$ irradiated as described above. After three washes with PBS containing $1 \mathrm{mM}$ $\mathrm{MgCl}_{2}$ and $1 \mathrm{mM} \mathrm{CaCl}_{2}\left(\mathrm{PBS}^{++}\right)$cells were grown for different time periods or immediately fixed with $3 \%(\mathrm{w} / \mathrm{v})$ PFA containing $2 \%$ Sucrose in $\mathrm{PBS}^{++}$for $15 \mathrm{~min}$ at room temperature. Indirect immunofluorescence was performed as already described [5]. Rabbit anti- $\gamma$ H2AX (1:100; Cell Signaling) was used as primary antibody and donkey anti-rabbit Alexa Fluor-555 (1:400; Invitrogen) as secondary antibody. Cell nuclei were stained with $0.1 \mu \mathrm{g} / \mu \mathrm{l}$ DAPI for $1 \mathrm{~min}$. Analysis was performed with LSM 510 META confocal laser scanning microscope (Carl Zeiss) and images was processed using ImageJ Software.

\subsection{Statistical data analysis}

Statistically significant differences between data sets were determined using unpaired Student's $t$-test (GraphPad Software, Inc.). Significance was attributed at the $95 \%$ level of confidence (Pvalue $<0.05)$.

\section{Results}

\subsection{Overexpression of sialidase NEU3 increases the radioresistance potential of U87MG cells}

To investigate whether NEU3 plays a role in determining radioresistance features in glioblastoma, U87MG cells were stably transfected with the murine form of NEU3 tagged with the HA epitope at the C-terminus (NEU3-HA). Expression of NEU3 was analyzed by means of enzymatic activity and Western-blot, in comparison to U87MG cells stably transfected with the empty vector (Mock). For this work we chose clone 1D1, showing a sialidase specific activity of $21.1 \pm 1.08 \mathrm{nmol} \mathrm{h}^{-1} \mathrm{mg}^{-1}$ which corresponds to an increase in the enzymatic activity of 21 -fold compared to Mock cells (Fig. 1A). Moreover, a specific band was detected with anti-HA antibody only in the cell extracts deriving from NEU3 transfected cells (Fig. 1A).

Mock and 1D1 cells were irradiated at different doses and subjected to clonogenic assay. After irradiation Mock cells showed a decreased number of colonies that was 63\% and 25\% after 2 and 4 Gy treatment, respectively, and compared to non-irradiated Mock cells (Fig. 1B). At all considered irradiation doses, 1D1 cells showed a statistically significant higher surviving potential compared to Mock cells, with a relative number of colonies that was $77 \%$ and $51 \%$ after 2 and $4 \mathrm{~Gy}$ treatment, respectively, and compared to nonirradiated 1D1 cells. These results demonstrate that expression of NEU3 confers higher radioresistance characteristics to U87MG cells after irradiation. 


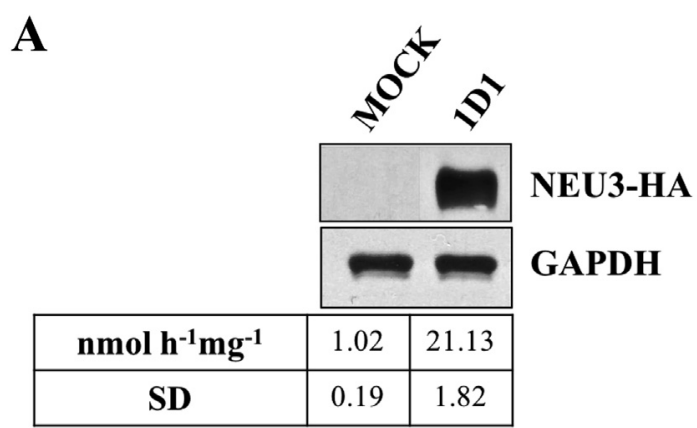

B

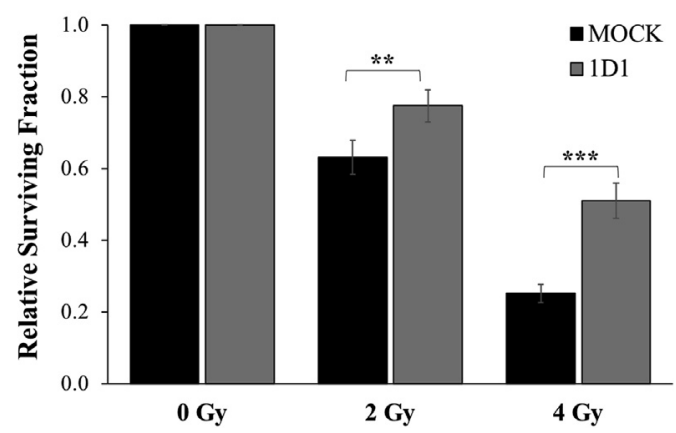

Fig. 1. Overexpression of sialidase NEU3 in U87MG cells increases cell survival after irradiation. (A) Analysis of the expression of sialidase NEU3 by means of Western blot (upper panel) and enzymatic activity (lower panel) in cell extracts deriving from Mock transfected (Mock) and NEU3 transfected U87MG cells (1D1). Values represent the average of five independent determinations. SD: Standard deviation. (B) Histogram showing the surviving fraction of Mock and 1D1 cells subjected to the indicated irradiation doses. Values represent the number of colonies counted relative to the corresponding not irradiated cells. Values represent the average of three independent experiments ( $\left.{ }^{* *} \mathrm{p}<0.01 ;{ }^{* * *} \mathrm{p}<0.001\right)$.

\subsection{The increased survival capacity of U87MG cells overexpressing sialidase NEU3 is driven by PI3K}

Ionizing radiations are known to activate EGFR [20,21] resulting in the further activation of down-stream survival pathways, including $E K_{1 / 2}$ and Akt pathways [22]. We analyzed this aspect in our cellular model and found that at 0 Gy U87MG cells expressing NEU3 showed a higher phosphorylation of $\mathrm{ERK}_{1 / 2}$, Akt-Thr308 and Akt-Ser473 compared to Mock cells (Suppl. Fig. 1). Irradiation increased the phosphorylation of $\mathrm{ERK}_{1 / 2}$ in both cells types, with a stronger effect in 1D1 cells. A similar behavior was observed for Akt-Thr308 while no significant changes were observed for AktSer473. Of note, the phosphorylation state of Akt-Ser473 in cells overexpressing NEU3 was always higher compared to Mock cells. These data demonstrate that overexpression of NEU3 in U87MG cells results in an increased basal activation of ERK $\mathrm{E}_{1 / 2}$ and Akt, and irradiation enhances this effect. Since $E K_{1 / 2}$ and Akt are downstream targets of EGFR, Mock and 1D1 cells were pre-treated or not with the Tyrosine Kinase inhibitor Erlotinib or with the chimeric EGFR monoclonal antibody Cetuximab before irradiation at the single dose of $2 \mathrm{~Gy}$ and subjected to clonogenic assay. Treatment with the two EGFR inhibitors alone resulted in a slight but similar decrease in the colony formation potential in both cell lines (Fig. 2A). Pre-treatment with Cetuximab or Erlotinib before irradiation did not modify the survival potential of 1D1 cells. On the contrary, the survival potential of Mock cells resulted significantly decreased after pre-treatment with the two EGFR inhibitors. These data demonstrate EGFR is not involved in the increased
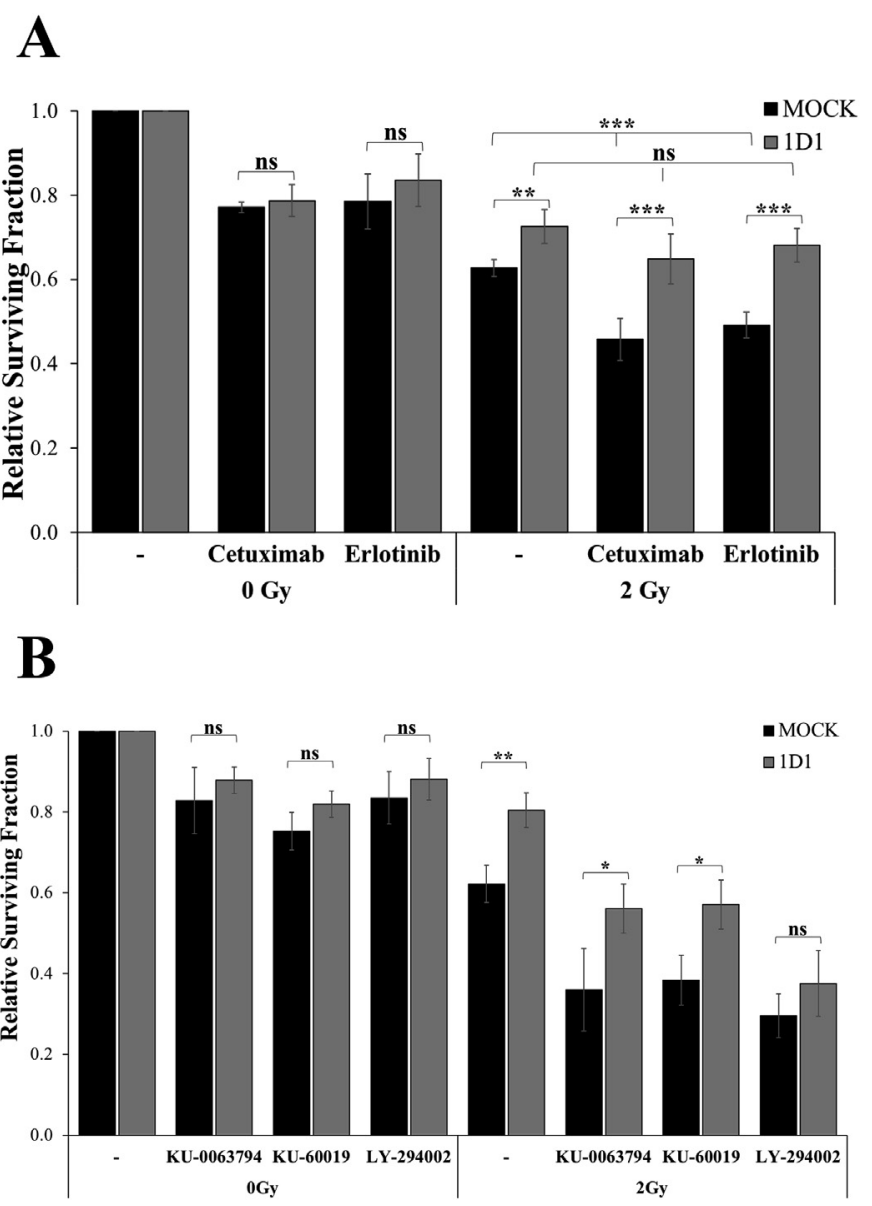

Fig. 2. The survival capacity of U87MG cells overexpressing sialidase NEU3 after irradiation is driven by PI3K. Mock and 1D1 cells were treated or not with (A) Cetuximab or Erlotinib as EGFR inhibitors, (B) KU-0063794 as mTORC1/2 inhibitor, KU60019 as ATM inhibitor, LY-294002 as PI3K inhibitor before irradiation. Values represent the number of colonies counted relative to the corresponding not irradiated cells. Values represent the average of three independent experiments $\left({ }^{*} \mathrm{p}<0.05 ;{ }^{* *} \mathrm{p}<0.01\right.$; **** $\mathrm{p}<0.001)$.

radioresistance features observed in U87MG cells expressing NEU3.

We then decided to inhibit the Akt pathway focusing on the two essential phosphorylated positions of the protein, i.e. Serine 473 and Threonine 308. As first, we blocked the Akt activation on Seine 473 by inhibiting mTORC2 and ATM protein kinase. Cells were pretreated or not with the mTORC1/2 inhibitor KU-0063794 or with the ATM inhibitor KU-60019 before irradiation and subjected to clonogenic assay. Pre-treatment with the inhibitors alone resulted in a similar decrease in the colony formation potential in both cell lines and compared to the corresponding untreated cells (Fig. 2B). After irradiation, the surviving potential of 1D1 cells after pretreatment with KU-0063794 and KU-60019 was statistically significant higher $(\mathrm{p}<0.05)$ compared to Mock cells. These data point out that inhibition of mTORC and ATM is not sufficient to abolish the radioresistance capacity observed in NEU3 expressing cells. We then blocked the activation of Atk at Threonine 308 by inhibiting PI3K, another key molecule in this signaling pathway. Interestingly, no statistically significant differences in the surviving fraction between Mock and 1D1 cells could be observed after irradiation in presence of the PI3K inhibitor (Fig. 2B). In detail, the surviving fraction observed in Mock and 1D1 cells after 2 Gy irradiation in presence of LY-294002 was $30 \%$ and $38 \%$, respectively, and compared to untreated and non-irradiated corresponding cells. 
These results demonstrate that PI3K is deeply involved in the higher surviving potential observed in U87MG cells expressing NEU3.

\subsection{Overexpression of sialidase NEU3 in U87MG cells increases the DNA-repair capacity}

An immediate effect of ionizing radiations is represented by the DNA damage, to which cells respond activating different DNArepair mechanisms. Among these, phosphorylation of histone $\operatorname{H2AX}(\gamma \mathrm{H} 2 \mathrm{AX})$ is a commonly used parameter to bring to evidence DNA double strand brakes (DNA-DSB) [23]. We investigated whether overexpression of NEU3 could influence the mechanisms of DNA-DSB after irradiation by the detection of $\gamma \mathrm{H} 2 \mathrm{AX}$-positive nuclei after irradiation. Without irradiation, no significant differences in the number of $\gamma \mathrm{H} 2 \mathrm{AX}$-positive nuclei between Mock and 1D1 cells were found (Fig. 3). After 30 min from irradiation with 2 and $4 \mathrm{~Gy}$, both cell lines showed a significant increase in the relative number of $\gamma \mathrm{H} 2 \mathrm{AX}$-positive nuclei compared to the corresponding

A

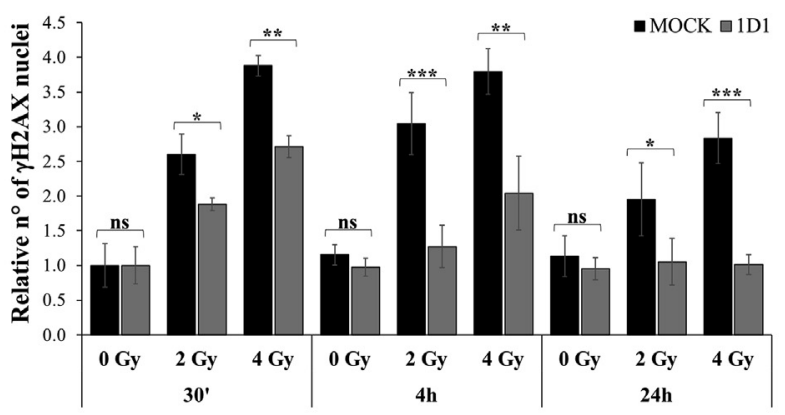

B

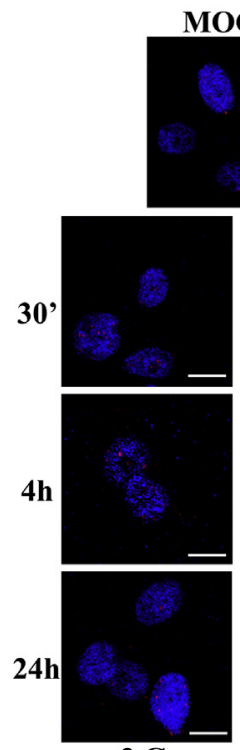

2 Gy
MOCK
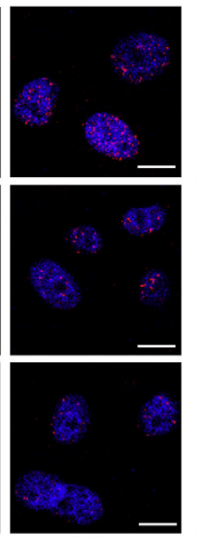

4 Gy
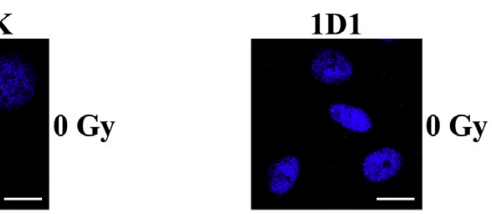

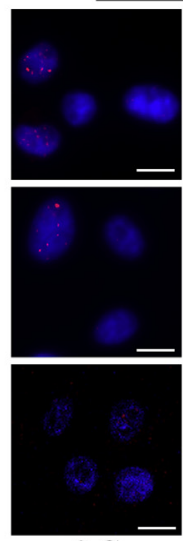

2 Gy
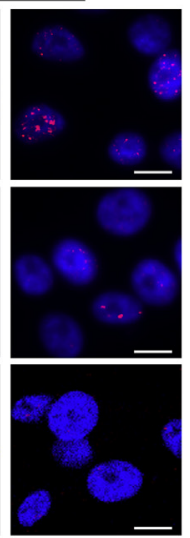

4 Gy

Fig. 3. Overexpression of sialidase NEU3 in U87MG cells increases the capacity of DNA repair. Mock and 1D1 cells were subjected to different irradiation doses and processed for indirect immunofluorescence for the detection of $\gamma \mathrm{H} 2 \mathrm{AX}$. (A) $\gamma \mathrm{H} 2 \mathrm{AX}$ positive nuclei were counted in 5 different fields, each with at least 20 nuclei, and the number related to the corresponding non-irradiated cells. Values represent the average of three independent experiments $\left({ }^{*} \mathrm{p}<0.05\right.$; ${ }^{* *} \mathrm{p}<0.01$; $\left.{ }^{* * *} \mathrm{p}<0.001\right)$. (B) Representative acquired images are shown. $\gamma \mathrm{H} 2 \mathrm{AX}$ labeling (red color), counterstained with DAPI (blue color). Scale bar: $10 \mu \mathrm{m}$. (For interpretation of the references to color in this figure legend, the reader is referred to the Web version of this article.)

non-irradiated cells. Although the relative number of $\gamma \mathrm{H} 2 \mathrm{AX}-\mathrm{pos}-$ itive nuclei increased in both cell lines in relation to the different radiation doses, a statistically significant lower number was observed in 1D1 cells. After $4 \mathrm{~h}$ from irradiation, the relative number of $\gamma \mathrm{H} 2 \mathrm{AX}$-positive nuclei in 1D1 was statistically lower compared to Mock cells. After $24 \mathrm{~h}$ from irradiation the relative number of $\gamma \mathrm{H} 2 \mathrm{AX}$-positive nuclei in 1D1 cells further decreased and remained significantly lower compared to Mock cell, reaching values comparable to non-irradiated 1D1 control cells. These data demonstrate that overexpression of NEU3 accelerates the DNA-DSB repair mechanisms of U87MG cells.

We then analyzed whether ATM, a protein with a key role in DNA-DSB repair, could be responsible for the different response to radiation-induced DNA damage observed in cells overexpressing NEU3. Mock and 1D1 cells were pre-treated or not with the ATM inhibitor KU-60019 before irradiation and after $24 \mathrm{~h} \gamma \mathrm{H} 2 \mathrm{AX}$-positive nuclei were counted. In Mock cells pre-treated with KU-60019 the number of $\gamma \mathrm{H} 2 \mathrm{AX}$-positive nuclei was 3-fold and 4-fold higher after $2 \mathrm{~Gy}$ and $4 \mathrm{~Gy}$ irradiation, compared to non-irradiated cells. The difference in the number of $\gamma \mathrm{H} 2 \mathrm{AX}$-positive nuclei between Mock and 1D1 cells in presence of ATM inhibitor was not statistically significant at $2 \mathrm{~Gy}$ and slightly significant at $4 \mathrm{~Gy}$ (Fig. 4). These results demonstrate that inhibition of ATM could counteract the protective effect on DNA-DSB repair induced by NEU3.

\section{Discussion}

In the last years, many reports demonstrated that upregulation of sialidase NEU3 occurs in different tumors [10-12]. GBM

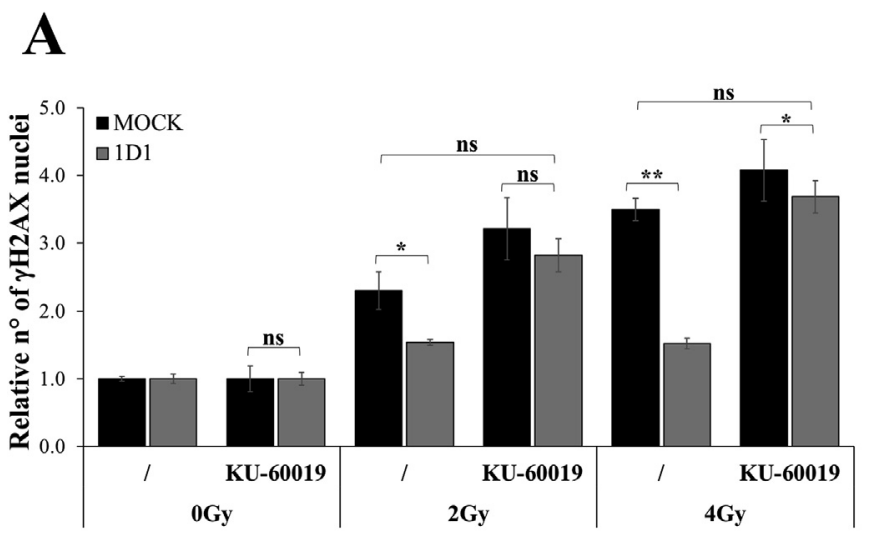

B

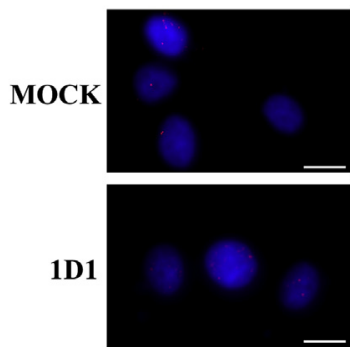

0 Gy

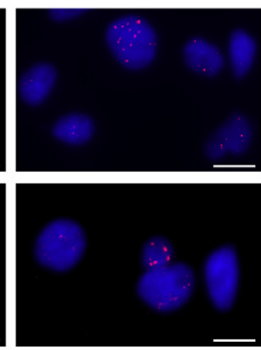

2 Gy
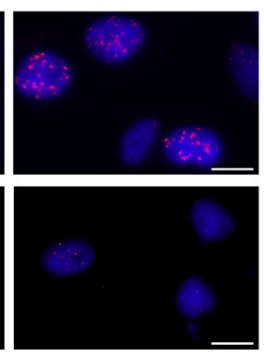

4 Gy

Fig. 4. The increased capacity of DNA repair in NEU3 overexpressing cells is driven by ATM. (A) Mock and 1D1 cells were treated or not with KU-60019 (ATM inhibitor) before irradiation and processed for indirect immunofluorescence for the detection of $\gamma \mathrm{H} 2 \mathrm{AX}$. Values represent the average of two independent experiments ${ }^{*} \mathrm{p}<0.05$; ${ }^{* *} \mathrm{p}<0.01$ ). (B) Representative acquired images are shown. $\gamma \mathrm{H} 2 \mathrm{AX}$ labeling (red color), counterstained with DAPI (blue color). Scale bar: $10 \mu \mathrm{m}$. (For interpretation of the references to color in this figure legend, the reader is referred to the Web version of this article.) 
represents the most common and most aggressive variant among primary brain tumors and radiotherapy is an important component of multimodality GBM treatment that comprehends also surgery and chemotherapy [18].

Ionizing radiations have been shown to induce EGFR activation [21] and Akt activation in multiple cell types, including some human GBM cells [24]. Both pathways are related to cell proliferation and cell survival features of tumor cells. Moreover, Akt regulates also DSB repair [25], and modifications in PI3K/Akt pathway can lead to radioresistance [26]. Akt is fully activated through phosphorylation of two key residues, i.e. Threonine 308 and Serine 473. In the cytosol, mTORC2 mediates phosphorylation at Serine 473 while PDK1 phosphorylates Akt at Threonine 308. The completely active Akt can translocate to the nucleus $[27,28]$. On the other hand, partially activated Akt can translocate to the nucleus after irradiation and become fully activated by nuclear PDK1 acting on Threonine 308 or by nuclear DNA-PKs, including ATM, at Serine 473 in response to DNA damage [29,30]. Moreover, beside phosphorylation of Akt at Serine 473, ATM is responsible for the phosphorylation of histone H2AX by ATM on Serine 139 ( $\gamma \mathrm{H} 2 \mathrm{AX}$ ), one of the earliest events involved in DNA Damage Response (DDR) after irradiation [23,29,31].

Little is known about the possible role played by NEU3 in relation to brain tumors such as GBM. A recent report demonstrated that overexpression of NEU3 in different GBM cell models, namely U251, A172 and T89G, reduced invasion and migration properties by promoting the assembly of focal adhesions, although the molecular mechanism remains unclear at present [32]. We investigated the possible role played by NEU3 in relation to radioresistance features in a GBM cell model represented by U87MG cells. Overexpression of NEU3 resulted in a significant increased capacity of U87MG to resist to irradiation. The radioresistance capacity of NEU3 expressing cells resulted to be independent from EGFR. Instead, by selective inhibition of mTORC, ATM and PDK1 we found that the PI3K/Akt pathway is deeply involved in increasing radioresistance features in U87MG cells overexpressing NEU3. A faster DNA-DSB repair was observed in cells overexpressing NEU3 that could be partially abolished by inhibition of ATM. Our findings represent the first evidence of new role played by sialidase NEU3 in tumor biology and open new perspectives for the pharmacological approaches and radiation therapy for glioblastoma.

\section{Funding}

This work was supported by the Italian Ministero dell'Istruzione, dell'Università e della Ricerca as ex-60\% funds to R.B. and E.M.; F.O and A.C. were supported by the Associazione Amici dell'Istituto del Radio, Spedali Civili di Brescia. This work was partly supported by BIOMANE Grant from University of Brescia to R.B. and E.M.

\section{Appendix A. Supplementary data}

Supplementary data to this article can be found online at https://doi.org/10.1016/j.bbrc.2018.11.086.

\section{Transparency document}

Transparency document related to this article can be found online at https://doi.org/10.1016/j.bbrc.2018.11.086.

\section{References}

[1] T. Miyagi, K. Yamaguchi, Mammalian sialidases: physiological and pathological roles in cellular functions, Glycobiology 22 (2012) 880-896, https:/| doi.org/10.1093/glycob/cws057.

[2] E. Monti, T. Miyagi, Structure and function of mammalian sialidases, Top. Curr. Chem. (2012), https://doi.org/10.1007/128_2012_328.

[3] E. Giacopuzzi, R. Bresciani, R. Schauer, E. Monti, G. Borsani, New insights on the sialidase protein family revealed by a phylogenetic analysis in metazoa, PLoS One 7 (2012), https://doi.org/10.1371/journal.pone.0044193 e44193.

[4] E. Monti, E. Bonten, A. d'Azzo, R. Bresciani, B. Venerando, G. Borsani, et al., Sialidases in vertebrates: a family of enzymes tailored for several cell functions, Adv. Carbohydr. Chem. Biochem. 64 (2010) 403-479, https://doi.org/ 10.1016/S0065-2318(10)64007-3.

[5] G. Zanchetti, P. Colombi, M. Manzoni, L. Anastasia, L. Caimi, G. Borsani, et al., Sialidase NEU3 is a peripheral membrane protein localized on the cell surface and in endosomal structures, Biochem. J. 408 (2007) 211-219, https://doi.org/ 10.1042/BJ20070503.

[6] L. Paolini, F. Orizio, S. Busatto, A. Radeghieri, R. Bresciani, P. Bergese, et al., Exosomes secreted by HeLa cells shuttle on their surface the plasma membrane-associated sialidase NEU3, Biochemistry 56 (2017) 6401-6408, https://doi.org/10.1021/acs.biochem.7b00665.

[7] E. Monti, M.T. Bassi, N. Papini, M. Riboni, M. Manzoni, B. Venerando, et al., Identification and expression of NEU3, a novel human sialidase associated to the plasma membrane, Biochem. J. 349 (2000) 343-351.

[8] T. Wada, Y. Yoshikawa, S. Tokuyama, M. Kuwabara, H. Akita, T. Miyagi, Cloning, expression, and chromosomal mapping of a human ganglioside sialidase, Biochem. Biophys. Res. Commun. 261 (1999) 21-27, https://doi.org/ 10.1006/bbrc.1999.0973.

[9] N. Papini, L. Anastasia, C. Tringali, G. Croci, R. Bresciani, K. Yamaguchi, et al., The plasma membrane-associated sialidase MmNEU3 modifies the ganglioside pattern of adjacent cells supporting its involvement in cell-to-cell interactions, J. Biol. Chem. 279 (2004) 16989-16995, https://doi.org/10.1074/ jbc.M400881200.

[10] T. Miyagi, K. Takahashi, K. Shiozaki, K. Yamaguchi, M. Hosono, Plasma membrane-associated sialidase confers cancer initiation, promotion and progression, Adv. Exp. Med. Biol. 842 (2015) 139-145, https://doi.org/ 10.1007/978-3-319-11280-0_9.

[11] T. Miyagi, K. Takahashi, K. Hata, K. Shiozaki, K. Yamaguchi, Sialidase significance for cancer progression, Glycoconj. J. 29 (2012) 567-577, https://doi.org/ 10.1007/s10719-012-9394-1.

[12] T. Miyagi, K. Takahashi, K. Yamamoto, K. Shiozaki, K. Yamaguchi, Biological and pathological roles of ganglioside sialidases, Prog Mol Biol Transl Sci 156 (2018) 121-150, https://doi.org/10.1016/bs.pmbts.2017.12.005.

[13] D. Bonardi, N. Papini, M. Pasini, L. Dileo, F. Orizio, E. Monti, et al., Sialidase NEU3 dynamically associates to different membrane domains specifically modifying their ganglioside pattern and triggering Akt phosphorylation, PLoS One 9 (2014), https://doi.org/10.1371/journal.pone.0099405 e99405.

[14] A. Mozzi, M. Forcella, A. Riva, C. Difrancesco, F. Molinari, V. Martin, et al., NEU3 activity enhances EGFR activation without affecting EGFR expression and acts on its sialylation levels, Glycobiology 25 (2015) 855-868, https://doi.org/ $10.1093 /$ glycob/cwv026.

[15] K. Yamamoto, K. Takahashi, K. Shiozaki, K. Yamaguchi, S. Moriya, M. Hosono, et al., Potentiation of epidermal growth factor-mediated oncogenic transformation by sialidase NEU3 leading to Src activation, PLoS One 10 (2015), https://doi.org/10.1371/journal.pone.0120578 e0120578.

[16] C.W. Brennan, R.G.W. Verhaak, A. McKenna, B. Campos, H. Noushmehr, S.R. Salama, et al., The somatic genomic landscape of glioblastoma, Cell 155 (2013) 462-477, https://doi.org/10.1016/j.cell.2013.09.034.

[17] D.N. Louis, A. Perry, G. Reifenberger, A. von Deimling, D. Figarella-Branger, W.K. Cavenee, et al., The 2016 world health organization classification of tumors of the central nervous system: a summary, Acta Neuropathol. 131 (2016) 803-820, https://doi.org/10.1007/s00401-016-1545-1.

[18] R. Stupp, W.P. Mason, M.J. van den Bent, M. Weller, B. Fisher, M.J.B. Taphoorn, et al., Radiotherapy plus concomitant and adjuvant temozolomide for glioblastoma, N. Engl. J. Med. 352 (2005) 987-996, https://doi.org/10.1056/ NEJMoa043330.

[19] C. Adamson, O.O. Kanu, A.I. Mehta, C. Di, N. Lin, A.K. Mattox, et al., Glioblastoma multiforme: a review of where we have been and where we are going, Expet Opin. Invest. Drugs 18 (2009) 1061-1083, https://doi.org/10.1517/ 13543780903052764.

[20] K. Dittmann, C. Mayer, B. Fehrenbacher, M. Schaller, U. Raju, L. Milas, et al., Radiation-induced epidermal growth factor receptor nuclear import is linked to activation of DNA-dependent protein kinase, J. Biol. Chem. 280 (2005) 31182-31189, https://doi.org/10.1074/jbc.M506591200.

[21] M. Kiyozuka, T. Akimoto, M. Fukutome, A. Motegi, N. Mitsuhashi, Radiationinduced dimer formation of EGFR: implications for the radiosensitizing effect of Cetuximab, Anticancer Res. 33 (2013) 4337-4346.

[22] H.-F. Li, J.-S. Kim, T. Waldman, Radiation-induced Akt activation modulates radioresistance in human glioblastoma cells, Radiat. Oncol. 4 (2009) 43, https://doi.org/10.1186/1748-717X-4-43.

[23] P. Maier, L. Hartmann, F. Wenz, C. Herskind, Cellular pathways in response to ionizing radiation and their targetability for tumor radiosensitization, Int. J. Mol. Sci. 17 (2016) 102, https://doi.org/10.3390/ijms17010102.

[24] H.-F. Li, J.-S. Kim, T. Waldman, Radiation-induced Akt activation modulates radioresistance in human glioblastoma cells, Radiat. Oncol. 4 (2009) 43, https://doi.org/10.1186/1748-717X-4-43.

[25] W.P. Roos, A.D. Thomas, B. Kaina, DNA damage and the balance between survival and death in cancer biology, Nat. Rev. Canc. 16 (2016) 20-33, https:// 
doi.org/10.1038/nrc.2015.2.

[26] M. Toulany, H. Peter Rodemann, Potential of Akt mediated DNA repair in radioresistance of solid tumors overexpressing erbB-PI3K-Akt pathway, Transl. Cancer Res. 2 (2013) 190-202, https://doi.org/10.3978/j.issn.2218676X.2013.04.09.

[27] A. Toker, Akt signaling: a damaging interaction makes good, Trends Biochem. Sci. 33 (2008) 356-359, https://doi.org/10.1016/j.tibs.2008.05.003.

[28] W.J. Davis, P.Z. Lehmann, W. Li, Nuclear PI3K signaling in cell growth and tumorigenesis, Front Cell Dev Biol 3 (2015) 24, https://doi.org/10.3389/ fcell.2015.00024.

[29] J. An, Y.-C. Huang, Q.-Z. Xu, L.-J. Zhou, Z.-F. Shang, B. Huang, et al., DNA-PKcs plays a dominant role in the regulation of $\mathrm{H} 2 \mathrm{AX}$ phosphorylation in response to DNA damage and cell cycle progression, BMC Mol. Biol. 11 (2010) 18, https://doi.org/10.1186/1471-2199-11-18.

[30] M. Fraser, S.M. Harding, H. Zhao, C. Coackley, D. Durocher, R.G. Bristow, MRE11 promotes AKT phosphorylation in direct response to DNA doublestrand breaks, Cell Cycle 10 (2011) 2218-2232, https://doi.org/10.4161/ cc.10.13.16305.

[31] D.V. Firsanov, L.V. Solovjeva, M.P. Svetlova, H2AX phosphorylation at the sites of DNA double-strand breaks in cultivated mammalian cells and tissues, Clin. Epigenet. 2 (2011) 283-297, https://doi.org/10.1007/s13148-011-0044-4.

[32] K. Takahashi, S. Proshin, K. Yamaguchi, Y, Yamashita, R. Katakura, K. Yamamoto, et al., Sialidase NEU3 defines invasive potential of human glioblastoma cells by regulating calpain-mediated proteolysis of focal adhesion proteins, Biochim. Biophys. Acta 1861 (2017) 2778-2788, https://doi.org 10.1016/j.bbagen.2017.07.023. 